\title{
LA ROTACIÓN DE CULTIVOS Y LAS PROPIEDADES DE LA CÁSCARA DE ALMENDRA COMO SUSTRATO
}

\section{THE ROTATION OF CROPS AND THE PROPERTIES OF ALMOND SHELL AS SUBSTRATE}

\author{
Gabino A. Martínez Gutiérrez ${ }^{1^{*}}$, Yolanda D. Ortiz Hernández ${ }^{1}$, Miguel Urrestarazu Gavilán², Ma. del \\ Carmen Salas San Juán² y Cirenio Escamirosa Tinoco ${ }^{1}$
}

\begin{abstract}
${ }^{1}$ Centro Interdisciplinario de Investigación para el Desarrollo Integral Regional, Unidad Oaxaca, Instituto Politécnico Nacional. Hornos 1003 Indeco. Xoxocotlán, Oaxaca, Oax. Tel. y Fax 01 (951) 5170610 Ext. 82761. ²Departamento de Producción Vegetal, Universidad de Almería. La Cañada de San Urbano s/n. 04120, Almería España.
\end{abstract}

*Autor para correspondencia (megabinin@yahoo.es)

\section{RESUMEN}

La cáscara del fruto del almendro (Prunus dulcis L.) es un residuo orgánico de la industria de los frutos secos, con mínima utilización en los países mediterráneos. Con el propósito de utilizar este material como medio de cultivo para hortalizas, se estudió el efecto del tiempo de uso como sustrato en función de sus propiedades físicas y químicas. Los experimentos se hicieron en invernadero tipo parral. El tiempo de uso de la cáscara de almendra como sustrato, estuvo determinado por tres ciclos de tomate (Lycopersicon esculentum Mill.) y dos de melón (Cucumis melo L.) de 165 y 100 d respectivamente, cultivados en sacos de $25 \mathrm{~L}$. Para el análisis del sustrato se tomaron muestras de tres sacos de los sustratos reutilizados, por cada ciclo de cultivo. Durante los primeros $165 \mathrm{~d}$ de uso, el porcentaje en peso de partículas medianas (de 0.125 a $2 \mathrm{~mm}$ ) disminuyó, mientras que las gruesas (de 4 a $8 \mathrm{~mm}$ ) aumentaron. Hasta los 430 d de uso, tanto las partículas finas (menores a 0.125 $\mathrm{mm})$ como las muy gruesas $(8-16 \mathrm{~mm})$ se incrementaron. A través del tiempo todas las propiedades físicas variaron significativamente. La densidad aparente disminuyó en $0.28 \mathrm{~g} \mathrm{~cm}^{-3}$, mientras que el espacio poroso total aumentó en $27 \%$. La mojabilidad y la contracción aumentaron más de $100 \%$, de acuerdo con el intervalo óptimo. Las relaciones aire-agua también se modificaron y fue a los $430 \mathrm{~d}$ de uso cuando la mayoría de sus valores se ubicaron en el óptimo. Los valores de las propiedades físicas y químicas fueron mejores a través del tiempo y se ubicaron en el intervalo óptimo, de modo que dicho sustrato se puede utilizar hasta $695 \mathrm{~d}$.

Palabras clave: Prunus dulcis, sustratos orgánicos, cultivo sin suelo.

\section{SUMMARY}

The almond (Prunus dulcis L.) shell is an organic residue of the dry fruit industry, which is underutilized in Mediterranean countries. With the purpose of utilizing this material as a substrate for cultivation, a study of its changes over time, regarding its physical and physical-chemical properties, as well as its granulometric distribution, was performed. Experiments were conducted in a greenhouse setting. The period of experimentation lasted for three growing cycles of tomato plants (Lycopersicon esculentum Mill.) and two cycles of melon plants (Cucumis melo L.) which are 165 and 100 d respectively, both cultivated in $25 \mathrm{~L}$ sacks. In order to analyze the substrate, samples were taken from three sacks utilized for each growing cycle. During the first $165 \mathrm{~d}$ of use the percent of weight of medium particulates (from 0.125 to $2 \mathrm{~mm}$ ) decreased, while the thicket particulates (of 4 to $8 \mathrm{~mm}$ ) increased. Over time, all the physical properties varied significantly. The apparent density was reduced by $0.28 \mathrm{~cm}^{-3}$, while the total porous space was increased by $27 \%$. The humidity and compaction increased over $100 \%$ at its optimal level. The air to water ratio was also modified, and when 430 d of use had been reached, the majority of its values were functioning at an optimal level. The values of the physical and chemical properties improved over time to their optimal level, so that this substrate can be used up to $695 \mathrm{~d}$.

Index words: Prunus dulcis, organic substrate, soilless culture.

\section{INTRODUCCIÓN}

El cultivo del almendro (Prunus dulcis L.) es de importancia económica y ambiental en los países mediterráneos, porque su fruta conocida como almendra es parte de la industria de frutos secos y por ser considerado como árbol colonizador de tierras marginales con fuertes pendientes (Martínez, 2000). Cuando la fruta es procesada industrialmente para obtener la semilla comestible (nuez o almendra), se elimina el hueso o endocarpio, comúnmente llamado "cáscara", que representa $20 \%$ del peso total. Para los años 2002-2003, la FAO estimó una superficie mundial cultivada con almendro de $1500 \quad 000$ ha (FAO 2001-2004).

En España se cultivan 525000 ha que producen 225 $000 \mathrm{t}$ de fruta por año, de las cuales $45000 \mathrm{t}$ corresponden al subproducto "cáscara" (MAPA, 1992-2002). Este material se utiliza principalmente como combustible para el cocimiento de ladrillos y artesanías de cerámica, así 
como para el acolchado de parques y jardines (Abad et al., 2000). Por la cercanía con la región hortícola de Almería, donde existen de 4000 a 4500 ha bajo cultivo sin suelo (Urrestarazu y Salas, 2002), la cáscara puede ser una alternativa de sustrato o componente de sustratos para el cultivo de plantas en contenedor (Jiménez et al., 2000), como tambien lo son el polvo de coco (Konduru et al., 1999; Abad et al., 2002), la fibra de madera (Gruda y Schnitzler 2004a, b; Muro et al., 2005), la corteza de árboles forestales, los desechos de la industria del corcho (Verdonck, 1983) y el compost de los desechos de destilería (Bustamante et al., 2008).

Urrestarazu et al. (2004), al evaluar dos granulometrías de cáscara de almendra $100 \%$ puras (obtenida directamente de la trituradora sin haber sido utilizada) en la producción y calidad del fruto de melón (Cucumis melo L.) y tomate (Lycopersicon esculentum Mill.), en comparación con el sustrato lana de roca, no encontraron diferencias significativas entre ambas granulometrías, ni tampoco con la lana de roca. Sin embargo, se desconoce el comportamiento de las propiedades de la cáscara de almendra como sustrato durante la rotación de cultivos hortícolas en invernadero. Por tanto, de la cáscara de almendra y de otros materiales orgánicos alternativos es necesario conocer las propiedades físicas y químicas, su comportamiento en cultivos de importancia económica y el tiempo de duración en el contenedor (Verdonck, 1983; Abad et al., 2002; Abad et al., 2005). El objetivo del presente trabajo fue evaluar el efecto de la rotación de dos cultivos hortícolas, tomate y melón, sobre algunas propiedades físicas y químicas de la cáscara de almendra, a través del tiempo de uso.

\section{MATERIALES Y MÉTODOS}

La investigación se llevó a cabo Almería, España, con cáscara de almendra proporcionada por la empresa "Borges de Andalucía". Este material fue almacenado en un lugar seco y fresco para poder utilizarlo como testigo en cada ciclo de cultivo y sus propiedades físicas y químicas fueron analizadas por única vez al inicio del experimento. El tiempo de uso de la cáscara de almendra como sustrato se hizo de acuerdo con las estaciones de cultivos de la comarca agrícola de Almería, donde se alternan especies de ciclo corto (p. e., melón) y largo (p. e., tomate), y consistió en tres ciclos de cultivos de tomate (cvs. 'Daniela', 'Daniela' y 'Pitenza') y dos de melón (cvs. 'Gustal' y 'Yucatán').

El experimento se hizo en condiciones de invernadero tipo parral (Valera et al., 1999). Los contenedores fueron sacos de $25 \mathrm{~L}$ y se compararon dos tratamientos: 1) sustrato nuevo y puro (la cáscara que sale de la trituradora, sin tamizarla y sin haber sido utilizada), y 2) sustrato reutilizado de cáscara de almendra, proveniente de un cultivo de tomate con $165 \mathrm{~d}$ de uso; cada tratamiento tuvo tres repeticiones, y el diseño experimental fue de bloques completos al azar. Al Tratamiento 1 se le llamó el día cero de uso y se consideró como el testigo (en cada ciclo hubo un testigo). Los muestreos del sustrato reutilizado se realizaron al final de cada ciclo de cultivo en $6 \mathrm{~L}$ del correspondiente sustrato, obtenidos de tres sacos en tres lugares equidistantes a lo largo del contenedor ( $2 \mathrm{~L}$ por saco por ciclo) y posteriormente fue eliminado sin reincorporación del sustrato nuevo o reutilizado en los sacos para cada muestreo. Todas las determinaciones físicas y químicas se hicieron por triplicado. El Cuadro 1 muestra la alternancia de los cultivos evaluados y el tiempo de uso de los sustratos.

Cuadro 1. Tiempo de uso del sustrato cáscara de almendra en dos cultivos alternos de tomate y melón.

\begin{tabular}{ccc}
\hline Cultivo & Días & $\begin{array}{c}\text { Tiempo de uso } \\
\text { (días) }\end{array}$ \\
\hline Testigo $^{\dagger}$ & 0 & 0 \\
Tomate & $165^{\dagger \dagger}$ & 165 \\
Melón & 100 & 265 \\
Tomate & 165 & 430 \\
Melón & 100 & 530 \\
Tomate & 165 & 695 \\
\hline
\end{tabular}

$\dagger$ Corresponde al sustrato sin usar.

${ }^{\dagger}$ A partir de este tiempo inició la comparación de las propiedades físicas y químicas.

Como un ciclo de cultivo se considero: $165 \mathrm{~d}$ para tomate y 100 d para melón. Al final de cada ciclo se tomó una muestra para determinar las propiedades físicas y químicas y sus cambios en relación con el sustrato sin uso (testigo). Después de cada muestreo en cada ciclo, no se removió el sustrato ni se agregó material nuevo o reutilizado a los sacos de cultivo, porque no reflejarían el tiempo de uso del sustrato. El primer cultivo se estableció en febrero del 2001 y el último en septiembre del 2002, para concluir en febrero del 2003.

Las propiedades físicas determinadas fueron: distribución del tamaño de partícula (expresada como porcentaje en peso) y contracción de volumen después de secado a $105^{\circ} \mathrm{C}$, de acuerdo con Richards et al. (1986) y Martínez (1992); densidad real y aparente, espacio poroso total y capacidad de retención de agua por el método propuesto por De Boodt y Verdonck (1972); relaciones aire-agua, por el método de De Boodt et al. (1974); y mojabilidad después del secado a $40{ }^{\circ} \mathrm{C}$ hasta peso 
constante, con base en la norma australiana AS 3743Appendix A Australian Regulation (1993).

Las propiedades químicas determinadas fueron: materia orgánica por pérdida, mediante calcinación durante $4 \mathrm{~h}$ a $550{ }^{\circ} \mathrm{C}$; contenido de aniones $\left(\mathrm{Cl}^{-}, \mathrm{NO}_{3}^{-}, \mathrm{H}_{2} \mathrm{PO}_{4}^{-}\right.$y $\left.\mathrm{SO}_{4}{ }^{2-}\right)$ y cationes $\left(\mathrm{Ca}^{2+}, \mathrm{Mg}^{2+}, \mathrm{K}^{+}\right.$y Na$\left.{ }^{+}\right)$en extracto de saturación (Warncke, 1986), con un cromatógrafo de líquidos (Dionex®, Sunnyvale, CA) 2000 i/SP y un cromatógrafo AS4A, con columnas CS12 de cationes conforme al método descrito por Gil de Carrasco et al. (1994), ambos expresados en mmolc $\mathrm{L}^{-1}$; carbono orgánico y nitrógeno total, mediante un microanalizador Carlo Erba ${ }^{\circledR}$ Mod. ANA 1500; capacidad de intercambio de cationes (CIC), por la modificación del método de $\mathrm{BaCl}_{2}$-Trietanolamina ( $\mathrm{pH}$ 8.1) propuesta por Lax et al. (1986) y Abad et al. (2002); y la relación carbono/nitrógeno $(\mathrm{C} / \mathrm{N})$ como el cociente entre los contenidos de carbono y nitrógeno.

Se efectuaron análisis de varianza (ANOVA) para determinar la relación entre el tiempo de uso del sustrato con las propiedades físicas y químicas, y análisis de regresión a las variables: densidad real y aparente, mojabilidad, contracción, capacidad de aireación, agua fácilmente disponible, agua de reserva, materia orgánica, materia mineral y relación carbono nitrógeno. Cuando el ANOVA resultó significativo, se aplicó la prueba de rango múltiple de Newman-Keuls. Los análisis estadísticos y correlaciones se hicieron con el programa Statgraphics Plus 4 (Statistical Graphics Corp., 1999).

\section{RESULTADOS Y DISCUSIÓN}

El tiempo de uso de la cáscara de almendra como sustrato en los cultivos alternos de tomate y melón tuvo efecto $(\mathrm{P} \leq 0.01)$ en la distribución granulométrica de los diferentes tamaños de partícula (Cuadro 2). A los $165 \mathrm{~d}$ de uso el porcentaje en peso de partículas medianas (0.25-2 $\mathrm{mm})$ disminuyó drásticamente, mientras que las finas $(<0.125 \mathrm{~mm})$ y gruesas $(>2 \mathrm{~mm})$ aumentaron. Posterior a este tiempo y hasta los $430 \mathrm{~d}$, las partículas de tamaño medio $(0.25-2 \mathrm{~mm})$ aumentaron, al igual que las gruesas (2-16 mm), sólo a los $265 \mathrm{~d}$, para después disminuir. Durante todo este tiempo, las partículas finas $(<0.125 \mathrm{~mm})$ aumentaron al igual que las muy gruesas $(8-16 \mathrm{~mm})$, aunque estas últimas no mostraron diferencias significativas.

El incremento del porcentaje en peso de las partículas finas, probablemente se debió a diferencias térmicas en el sustrato que ocasionó la fracturación de partículas gruesas (4-8 mm) como lo mencionan Carmona et al. (2001) y al arrastre de partículas por el fertirriego, mientras que el ligero aumento de partículas muy gruesas $(8-16 \mathrm{~mm})$ se atribuye a la formación de conglomerados por adherencia entre las partículas de la cáscara de almendra y las raíces de las plantas de tomate y melón. Posterior a los 430 d de uso no se encontró una tendencia definida en el comportamiento del porcentaje de partículas para todos los tamaños estudiados, probablemente debido al muestreo realizado. El volumen de cada saco disminuyó a través del tiempo, pero no por efecto del muestreo sino por el peso de la planta y degradación de las partículas (Birian y Eliassaf, 1980). El equilibrio granulométrico entre los diferentes tamaños de partículas de la cáscara de almendra hasta los 430 d de uso, mejoró algunos valores de las propiedades físicas como lo encontrado en el sustrato perlita por Marfá et al. (1993) y en las propiedades químicas del sustrato fibra de coco por Noguera et al. (1997).

Todas las propiedades físicas presentaron cambios $(\mathrm{P} \leq 0.01)$ con el tiempo de uso de la cáscara de almendra como sustrato (Cuadro 3). La densidad real (DR) aumentó linealmente $\left(\mathrm{R}^{2}=0.92, \mathrm{P} \leq 0.01\right)$ mientras que la densidad aparente (DA) disminuyó $\left(\mathrm{R}^{2}=0.93, \mathrm{P} \leq 0.01\right)$. Sin embargo, los valores de ambas propiedades no superaron el intervalo óptimo (IO) de los sustratos de cultivo indicados por Abad et al. (1993). Cuando el sustrato cáscara de almendra fue nuevo, el espacio poroso total (EPT) no se ubicó en el óptimo y continuó disminuyendo durante los primeros $165 \mathrm{~d}$ de uso, para alcanzar su valor óptimo a los $265 \mathrm{~d}$. La capacidad de retención de agua (CRA) aumentó durante los primeros 165 d de uso; después de este tiempo no se encontraron diferencias significativas y los valores fueron inferiores al óptimo (Abad et al., 1993). El mejoramiento al paso del tiempo de los parámetros DR, DA y EPT, se debió a la mejor distribución granulométrica entre partículas finas y grandes; resultados similares fueron encontrados por Handreck (1983) para la corteza de pino y Konduru et al. (1999) para partículas finas de fibra de coco.

La capacidad de aireación (CA) disminuyó a los 165 d cuando se ubicó en el óptimo. A los $265 \mathrm{~d}$ el valor de esta propiedad casi se duplicó y posteriormente disminuyó, pero permaneció dentro del valor óptimo hasta los $530 \mathrm{~d}$. Este comportamiento estuvo fuertemente asociado con la variación del tamaño de partículas, que en este tiempo de uso $(530 \mathrm{~d})$ aumentaron las partículas finas $(<0.25 \mathrm{~mm})$ y medianas $(0.25-2.0 \mathrm{~mm}) \mathrm{y}$ disminuyeron las gruesas $(>2 \mathrm{~mm})$.

La mojabilidad y contracción de volumen aumentaron linealmente a través del tiempo. La primera a los $165 \mathrm{~d}$ de uso superó el óptimo, y la segunda lo alcanzó a los 695 d de uso. En ambos casos, se encontró una correlación significativa $(\mathrm{P} \leq 0.01)$ con respecto a los días de uso 
$\left(\mathrm{R}^{2}=0.93\right.$ y 0.96 , respectivamente). Este comportamiento afectó directamente a la rehidratación del material, como lo reportado por Carmona et al. (2001) al evaluar residuos de la industria del corcho.

Cuadro 2. Efecto del tiempo de uso del sustrato cáscara de almendra en cultivos alternos de tomate y melón, sobre la distribución granulométrica, en por ciento del peso.

\begin{tabular}{|c|c|c|c|c|c|c|c|c|}
\hline \multirow{3}{*}{$\begin{array}{c}\text { Tiempo de uso } \\
\text { (días) }\end{array}$} & \multicolumn{8}{|c|}{ Tamaño de partícula (mm) } \\
\hline & \multicolumn{2}{|c|}{ Finas } & \multicolumn{3}{|c|}{ Medianas } & \multicolumn{3}{|c|}{ Gruesas } \\
\hline & $<0.125$ & $0.125-0.25$ & $0.25-0.50$ & $0.5-1$ & $1-2$ & $2-4$ & $4-8$ & $8-16$ \\
\hline \multicolumn{9}{|c|}{ (\% en peso) } \\
\hline 0 & $0.1 \mathrm{c}^{\dagger}$ & $0.3 \mathrm{c}$ & $2.5 \mathrm{c}$ & $12.8 \mathrm{~b}$ & $33.9 \mathrm{~b}$ & $38.6 \mathrm{~b}$ & $10.4 \mathrm{~b}$ & $1.1 \mathrm{c}$ \\
\hline 265 & $0.5 \mathrm{c}$ & $0.9 \mathrm{c}$ & $2.4 \mathrm{c}$ & $5.0 \mathrm{c}$ & $22.0 \mathrm{~b}$ & $49.5 \mathrm{a}$ & $15.7 \mathrm{~b}$ & $3.5 \mathrm{~b}$ \\
\hline 430 & $1.3 \mathrm{a}$ & $3.5 \mathrm{a}$ & $5.9 \mathrm{~b}$ & $19.1 \mathrm{a}$ & $43.8 \mathrm{a}$ & $17.5 \mathrm{c}$ & $4.4 \mathrm{c}$ & $3.7 \mathrm{~b}$ \\
\hline 530 & $0.6 \mathrm{~b}$ & $1.7 \mathrm{~b}$ & $3.2 \mathrm{~b}$ & $5.2 \mathrm{~b}$ & $16.1 \mathrm{c}$ & $38.5 \mathrm{~b}$ & $19.9 \mathrm{a}$ & $13.9 \mathrm{a}$ \\
\hline
\end{tabular}

Cuadro 3. Propiedades físicas del sustrato cáscara de almendra en cultivos alternos de tomate y melón.

\begin{tabular}{|c|c|c|c|c|c|c|c|c|}
\hline \multirow{2}{*}{ Propiedad } & \multicolumn{7}{|c|}{ Tiempo de uso (días) } & \multirow{2}{*}{$\begin{array}{c}\text { Intervalo } \\
\text { óptimo (IO) }\end{array}$} \\
\hline & 0 & 165 & 265 & 430 & 530 & 695 & Significancia & \\
\hline Densidad aparente $\left(\mathrm{g} \mathrm{cm}^{-3}\right)$ & $0.4 \mathrm{a}$ & $0.3 \mathrm{~b}$ & $0.2 \mathrm{c}$ & $0.1 \mathrm{~d}$ & $0.1 \mathrm{~d}$ & $0.1 \mathrm{~d}$ & $* *$ & $<0.40^{(\dagger \dagger)}$ \\
\hline Mojabilidad (minutos) & $0.5 \mathrm{c}$ & $19.0 \mathrm{~b}$ & $15.0 \mathrm{~b}$ & $24.0 \mathrm{~b}$ & $37.8 \mathrm{ba}$ & $47.3 \mathrm{a}$ & $* *$ & $<5.00^{(\dagger \dagger+)}$ \\
\hline Contracción (\% v/v) & $9.9 \mathrm{~d}$ & $17.7 \mathrm{c}$ & $18.0 \mathrm{~b}$ & $18.2 \mathrm{~b}$ & $22.6 \mathrm{~b}$ & $31.8 \mathrm{a}$ & $* *$ & $<30.00^{(\dagger \dagger)}$ \\
\hline Capacidad de retención de agua $\left(\mathrm{ml} \mathrm{L}^{-1}\right)$ & $188.6 \mathrm{c}$ & $257.8 \mathrm{~b}$ & $256.9 \mathrm{~b}$ & $264.0 \mathrm{~b}$ & $253.7 \mathrm{~b}$ & $282.5 \mathrm{a}$ & $*$ & $600-1000^{(\dagger \dagger)}$ \\
\hline Agua de reserva $(\% \mathrm{v} / \mathrm{v})$ & $4.6 \mathrm{a}$ & $0.5 \mathrm{c}$ & $0.2 \mathrm{~d}$ & $0.7 \mathrm{c}$ & $1.3 \mathrm{~b}$ & $0.7 \mathrm{c}$ & $* *$ & $4-10^{(\dagger \dagger)}$ \\
\hline Agua total disponible (\% v/v) & $5.8 \mathrm{a}$ & $3.5 \mathrm{~b}$ & $2.7 \mathrm{c}$ & $3.2 \mathrm{~b}$ & $4.3 \mathrm{a}$ & $4.5 \mathrm{a}$ & $*$ & $24-30^{(\dagger \dagger)}$ \\
\hline Agua difícilmente disponible (\% v/v) & $29.2 \mathrm{~d}$ & $39.2 \mathrm{c}$ & $40.8 \mathrm{~b}$ & $54.5 \mathrm{~b}$ & $59.8 \mathrm{~b}$ & $72.8 \mathrm{a}$ & $* *$ & - \\
\hline
\end{tabular}


Las variaciones de humedad en los diferentes tiempos de uso de la cáscara de almendra se reflejaron en los perfiles de la curva de liberación de agua para cada sustrato estudiado (Figura 1). De 0 a $265 \mathrm{~d}$ de uso se observan tendencias similares representadas por una forma lineal hasta los $2 \mathrm{kPa}$; pero a tensiones superiores de $3 \mathrm{kPa}$ adquieren una tendencia asintótica. Comportamiento similar presentaron las curvas de liberación de agua de los materiales con 430 y 530 d de uso, mientras que el material con 695 d difirió notablemente de los demás. Hasta los $265 \mathrm{~d}$ de uso, el sustrato cáscara de almendra contenía $60 \%$ en volumen de aire y $40 \%$ de agua; posteriormente el proceso fue inverso al aumentar el contenido de agua y disminuir el de aire, fenómeno que fue más acentuado en el sustrato con $695 \mathrm{~d}$ de uso, tiempo en el cual se presentaron muertes de plantas de tomate por hipoxia radical, similar a lo mencionado por Abad et al. (2005) para el cultivo de hortalizas en contenedores de poca altura o profundidad.

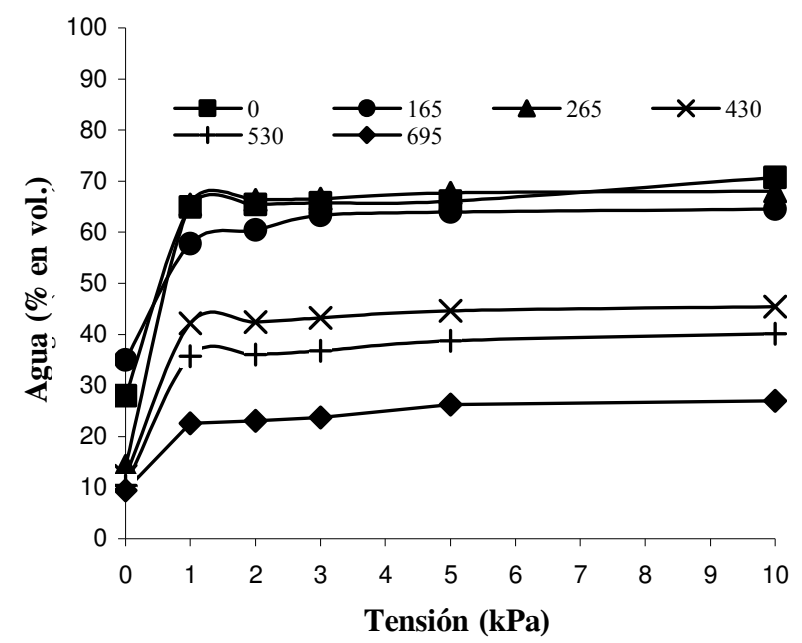

Figura 1. Curva de liberación de agua del sustrato cáscara de almendra, en función de la presión de succión para diferentes tiempos de uso en cultivos alternos de tomate y melón.

Los valores de las relaciones aire-agua fueron significativamente diferentes con respecto a los días de uso. El agua fácilmente disponible (AFD) aumentó linealmente en función a los días de uso del sustrato $\left(R^{2}=0.881 ; P \leq 0.05\right)$, pero no alcanzó a ubicarse en el óptimo. El agua de reserva (AR) decreció significativamente después del primer cultivo $\mathrm{y}$ posteriormente permaneció constante. Como resultado de la suma de las dos últimas propiedades, el agua total disponible mostró un comportamiento similar al agua de reserva, mientras que el agua difícilmente disponible se duplicó durante los primeros días de cultivo.
Todas las propiedades químicas (Cuadro 4) difieren $(\mathrm{P} \leq 0.01)$ con respecto a los días de uso de la cáscara de almendra como sustrato hortícola. El valor del $\mathrm{pH}$ fue ligeramente superior al óptimo, sin mostrar grandes cambios a través del tiempo. Posteriormente, a los $530 \mathrm{~d}$ de uso aumentó en 1.33 unidades, y éste fue el máximo valor alcanzado; este incremento posiblemente estuvo asociado con la frecuencia del fertirriego, como lo encontrado por Martínez y Abad (1992) en el sustrato zepiolita en el cultivo de tomate. Los valores de conductividad eléctrica (CE) se mantuvieron dentro del óptimo, con excepción del valor obtenido a los 430 d. Estos valores pueden afectar al crecimiento de ciertas plantas sensibles a salinidad (Bunt, 1988). Sin embargo, se ha demostrado que los altos valores de $\mathrm{pH}$ y $\mathrm{CE}$ del sustrato no presentan riesgo para su uso, ya que el programa de riego aplicado durante el desarrollo del cultivo provoca una eficaz lixiviación de las sales solubles en exceso (Noguera et al., 1997; Abad et al., 2002).

La capacidad de intercambio catiónico (CIC) de la cáscara de almendra nueva fue alta $\left(71.12 \mathrm{cmolc} \mathrm{kg}^{-1}\right)$, ligeramente superior a la mostrada por la fibra de coco de origen tailandés (70.4 cmolc $\left.\mathrm{kg}^{-1}\right)$ (Abad et al., 2002) e inferior a la turba (Sphagnum spp.), la cual alcanza valores

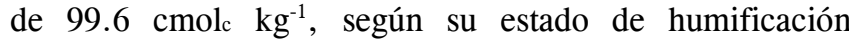
(Puustjarvi y Robertson, 1975). Pero al utilizarse por primera vez $(165$ d) como sustrato, disminuyó considerablemente y luego se mantuvo hasta los $695 \mathrm{~d}$ entre 44.82 y $37.54 \mathrm{cmolc}_{\mathrm{kg}}^{-1}$. Estos resultados son similares a los encontrados en fibra de coco por Arenas et al. (2002). Contrario a los resultados obtenidos, Puustjarvi y Robertson (1975) y Puustjarvi (1994) indican que el proceso de descomposición de los materiales orgánicos incrementa la CIC.

Posiblemente la disminución de la CIC del sustrato cáscara de almendra a través del tiempo de uso estuvo asociada con el método analítico utilizado, el cual se aplica en suelos y fue modificado para materiales orgánicos susceptibles de utilizarse como sustratos. La materia orgánica $(\mathrm{MO})$ de la cáscara de almendra nueva $(0 \mathrm{~d})$ fue alta, disminuyó paulatinamente con el tiempo, sin superar el intervalo óptimo ( $>80 \%$ MS) y mostró alta correlación lineal $\left(\mathrm{R}^{2}=0.925 ; \mathrm{P} \leq 0.05\right)$. Resultado similar fue encontrado para la corteza de pino y viruta de madera por Ansorena (1994), en materiales orgánicos nuevos y reutilizados en un ciclo de cultivo; este mismo autor menciona que en el compostaje natural o inducido, los materiales orgánicos experimentan una serie de cambios en su composición química, hasta alcanzar una cierta estabilidad biológica, contrario a lo que le ocurre a la turba Sphagnum que al ser sometida durante largos tiempos a los procesos naturales de degradación biológica 
no muestra un proceso de descomposición en corto tiempo y su estabilidad es alta.

La relación carbono nitrógeno $(\mathrm{C} / \mathrm{N})$ como indicador del tipo de materia orgánica, su madurez y estabilidad, mostró diferencias $(\mathrm{P} \leq 0.01)$ entre días de uso. La cáscara de almendra sin utilizar tuvo el máximo valor (172.83) que superó el óptimo, lo cual puede repercutir en la inmovilización del nitrógeno (Abad et al., 2002; Arenas et $a l ., 2002)$ si se utiliza este material como medio de cultivo para la producción de plantas en contenedor. Sin embargo, con el uso como sustrato dicho valor decreció desde los primeros $165 \mathrm{~d}$ y se mantuvo entre 10.42 y 19.40 , valores que están dentro del óptimo. Esto indica que a diferencia del material nuevo poco descompuesto, con la reutilización la materia orgánica alcanzó su madurez y estabilidad química mediante la humificación (Burés, 1997; Bernal et al., 1998), y muestra una relación cuadrática con respecto a los días de uso $\left(\mathrm{R}^{2}=0.84 ; \quad \mathrm{P} \leq 0.05\right)$. Esta disminución de los valores de la relación $\mathrm{C} / \mathrm{N}$ a través del tiempo de uso, también fue encontrado por Ansorena (1994), en los sustratos corteza de pino y viruta de madera, en comparación con la turba, y por Herrera et al. (2008) en el compost de desechos municipales.

El contenido y comportamiento de macroelementos (mmolc $\mathrm{L}^{-1}$ ) estuvo marcadamente representada por dos grupos de sustratos de cáscara de almendra con respecto al contenido de elementos asimilables y a la edad de uso como medio de cultivo (Cuadro 5). El primer grupo corresponde de 0 a $265 \mathrm{~d}$, en el cual los iones $\mathrm{Ca}^{2+}, \mathrm{K}^{+}$, $\mathrm{Cl}^{-}$y $\mathrm{NO}_{3}^{-}$mostraron valores inferiores a la cáscara nueva (0 d de uso), mientras que $\mathrm{Mg}^{2+}, \mathrm{Na}^{+}$y $\mathrm{H}_{2} \mathrm{PO}_{4}^{-}$fueron superiores. El segundo grupo correspondió de los 430 a los $695 \mathrm{~d}$ de uso, en donde todos los valores fueron superiores al de la cáscara nueva, con excepción del $\mathrm{Cl}^{-}$ que fue inferior. Al igual que en el primer grupo, la mayoría de estos macro-elementos no rebasaron el óptimo. Este aumento de los nutrimentos solubles en los materiales reutilizados pudo estar asociado con la fertirrigación intermitente y abundancia de partículas finas (menores a $0.5 \mathrm{~mm}$ ), la cual se ha demostrado que favorece la presencia de elementos a medida que disminuye el tamaño de partícula (Noguera et al., 1997).

Cuadro 4. Propiedades químicas del sustrato cáscara de almendra en cultivos alternos de tomate y melón.

\begin{tabular}{|c|c|c|c|c|c|c|c|c|}
\hline \multirow[b]{2}{*}{ Propiedad } & \multicolumn{7}{|c|}{ Tiempo de uso (días) } & \multirow{2}{*}{$\begin{array}{l}\text { Intervalo } \\
\text { óptimo } \\
\text { (IO) }\end{array}$} \\
\hline & 0 & 165 & 265 & 430 & 530 & 695 & Significancia & \\
\hline $\mathrm{pH}$ & $5.0 \mathrm{c}^{\dagger}$ & $6.0 \mathrm{~b}$ & $6.9 \mathrm{~b}$ & $6.6 \mathrm{~b}$ & $7.9 \mathrm{a}$ & $6.2 \mathrm{~b}$ & ** & $5.5-6.3^{\dagger}$ \\
\hline Conductividad eléctrica $\left(\mathrm{dS} \mathrm{m}^{-1}\right)$ & $2.4 \mathrm{~b}$ & $2.2 \mathrm{~b}$ & $2.0 \mathrm{c}$ & $3.9 \mathrm{a}$ & $3.1 \mathrm{a}$ & $2.9 \mathrm{~b}$ & $* *$ & $2.0-3.50^{\dagger}$ \\
\hline $\begin{array}{l}\text { Capacidad de intercambio catiónico (cmolc } \\
\mathrm{kg}^{-1} \text { ) }\end{array}$ & $71.1 \mathrm{a}$ & $44.8 \mathrm{~b}$ & $32.8 \mathrm{~b}$ & $31.9 \mathrm{~b}$ & $32.8 \mathrm{~b}$ & $37.5 \mathrm{~b}$ & $* *$ & $>20^{\dagger}$ \\
\hline Materia orgánica $(\%, \mathrm{MS})$ & $99.0 \mathrm{a}$ & $95.0 \mathrm{~b}$ & $95.8 \mathrm{~b}$ & $92.7 \mathrm{~b}$ & $87.3 \mathrm{c}$ & $85.6 \mathrm{c}$ & $* *$ & $>80^{\dagger}$ \\
\hline Relación C/N & $172.8 \mathrm{a}$ & $19.4 \mathrm{~b}$ & $10.3 \mathrm{~d}$ & $20.9 \mathrm{~b}$ & $10.4 \mathrm{~d}$ & $15.8 \mathrm{c}$ & ** & $20-40^{\dagger \dagger}$ \\
\hline
\end{tabular}

** Altamente significativo al nivel de probabilidad de 0.01. IO = Intervalo óptimo de acuerdo con ${ }^{(\dagger)} \mathrm{Abad}$ et al. (1993) y ${ }^{(\dagger \dagger)} \mathrm{Abad}$ et al. $(2000) .{ }^{\dagger}$ Medias con letras iguales entre filas no son estadísticamente diferentes (Newman-Keuls, 0.05).

Cuadro 5. Contenido de aniones y cationes del sustrato cáscara de almendra en cultivos alternos de tomate y melón.

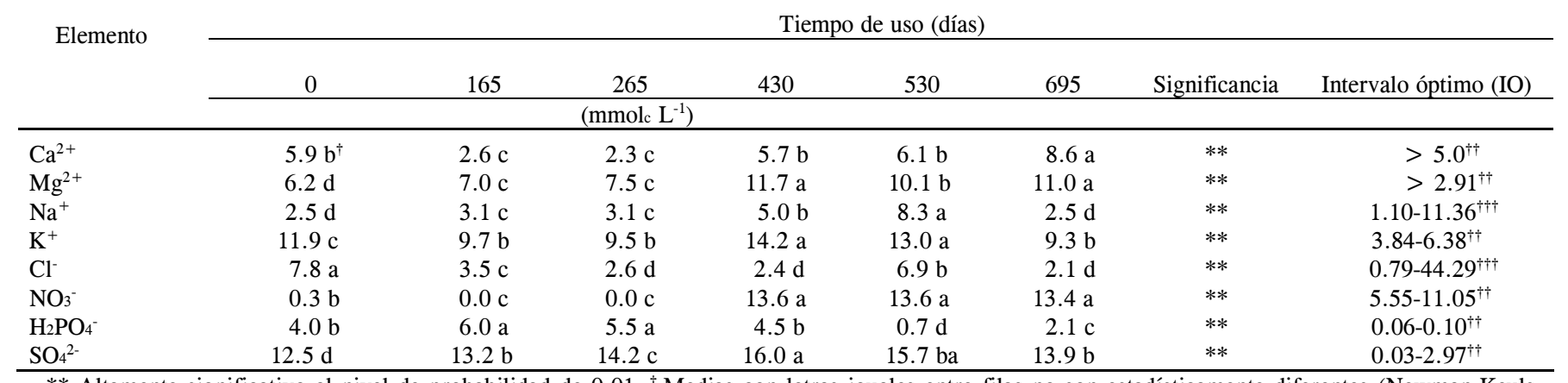

** Altamente significativo al nivel de probabilidad de $0.01 .{ }^{\dagger}$ Medias con letras iguales entre filas no son estadísticamente diferentes (Newman-Keuls, 0.05). IO = Intervalo Optimo de acuerdo con ${ }^{(\dagger+)}$ Abad et al. (1993) y ${ }^{(\dagger+)}$ Abad et al. (2000). 



\section{CONCLUSIONES}

El tiempo de uso de la cáscara de almendra como sustrato en cultivos hortícolas modificó la distribución granulométrica del tamaño de partículas. Durante los primeros $165 \mathrm{~d}$ de uso, las partículas de tamaño medio $(0.25$ a $2 \mathrm{~mm})$ disminuyeron, mientras que las finas (menor a $0.125 \mathrm{~mm})$, gruesas $(2-8 \mathrm{~mm})$ y muy gruesas $(8-$ $16 \mathrm{~mm}$ ) se incrementaron. Posterior a este tiempo y hasta los $430 \mathrm{~d}$ de uso, todos los tamaños aumentaron, con excepción de las partículas gruesas $(2-8 \mathrm{~mm})$ que disminuyeron. Después de los 430 d de uso el comportamiento granulométrico no mostró una tendencia definida. Esto repercutió en el aumento de los valores del espacio poroso total, capacidad de retención de agua, mojabilidad y contracción de volumen. Estas dos últimas propiedades, al paso del tiempo se convierten en no viables para su utilización como medios de cultivo. Las relaciones aire-agua también se modificaron, y fue a los $430 \mathrm{~d}$ de uso cuando la mayoría de sus valores se ubicaron en el nivel óptimo

A través del tiempo, todas las propiedades físicas y químicas mejoraron sus valores y algunas se ubicaron en el nivel óptimo. El comportamiento nutricional de la cáscara de almendra reutilizada, aumentó ligeramente después de los $430 \mathrm{~d}$ de uso.

Finalmente, la cáscara de almendra se puede utilizar hasta 695 d como sustrato orgánico en cultivos alternos de tomate y melón, siempre y cuando se tenga especial cuidado en la mojabilidad y contracción de volumen. Para la primera característica deberá mantenerse húmedo el sustrato, y para la segunda conviene agregar material nuevo al término de cada ciclo de cultivo.

\section{BIBLIOGRAFÍA}

Abad M, F Fornes, C Carrion, P Noguera, V Noguera, A Maquieira, R Puchades (2005) Physical properties of various coconut coir dusts compared to peat. HortScience 40:2138-2144.

Abad M, P Noguera, R Puchades, A Maquieira, V Noguera (2002) Physical-chemical and chemical properties of some coconut coir dusts for use as a peat substitute for containerised ornamental plants. Biores. Technol. 82:241-245.

Abad M, P Noguera, S Burés (2000) Inventario de sustratos y materiales para ser utilizados como sustratos o componentes de sustratos en España. Acta Hort. 32:361-377.

Abad M, P F Martínez, M D Martínez, J Martínez (1993) Evaluación agronómica de los sustratos de cultivo. Acta Hort. 11:141-154.

Ansorena M J (1994) Sustratos. Propiedades y Caracterización. MundiPrensa. $172 \mathrm{p}$.

Arenas M, C S Vavrina, J A Cornell, E A Hanlon, G J Hochmuth (2002) Coir as an alternative to peat in media for tomato transplant production. HortScience 37:309-312.
Australian Standard 3743-Appendix A (1993) Australian Standard for Potting Mixes. 2nd ed. Standards Association of Australia. Homebush, NSW, Australia. 320 p.

Bernal M P, C Paredes, A Sánchez-Monedero, J Cegarra (1998) Maturity and stability parameters of composts prepared with a wide range of organic wastes. Biores. Technol. 63:91-93.

Birian I, A Eliassaf (1980) The effect of container size and aeration condition on growth of roots and canopy of woody plants. Sci. Hort. 12:385-394.

Bunt A C (1988) Media and Mixes for Container-Grown Plants. 2nd ed. Unwin Hyman Ltd, London. $350 \mathrm{p}$.

Burés S (1997) Sustratos. Ed. Agrotécnicas. S. L. España. 342 p.

Bustamante M A, C Paredes, R Mora, E Agullo. M D Pérez-Murcia, M Abad (2008) Composts from distillery wastes as peat substitutes for transplant production. Resources Conservation and Recycling 52:792-799.

Carmona C H E, A J Ordovas, A M Moreno, A M Aguado, M C Ortega de la T (2001) Reciclado de los residuos de la industria del corcho para su aprovechamiento como sustrato de cultivo. Consejería de Agricultura y Pesca. Junta de Andalucía, España. 36 p.

De Boodt M, O Verdonck, I Cappaert (1974) Method for measuring the water release curve of organic substrates. Acta Hort. 37:20542062

De Boodt M, O Verdonck (1972) The physical properties of the substrates in horticulture. Acta Hort. 26:37-44.

FAO (2001-2004) Agricultural Data. FAOSTAT. Roma. http://faostat.fao.org/

Gil de Carrasco C, M Guzmán, F A Lorente, M Urrestarazu (1994) Xylem sap extraction: A method. Comm. Soil Sci. Plant Anal. 27:1859-1874.

Gruda N, W H Schnitzler (2004a) Suitability of wood fiber substrate for production of vegetable transplants I. Physical properties of wood fiber substrates. Sci. Hort. 100:309-322.

Gruda N, W H Schnitzler (2004b) Suitability of wood fiber substrate for production of vegetable transplants II. The effect of wood fiber substrates and their volume weights on the growth of tomato transplants. Sc. Hort. 100:333-340.

Handreck K A (1983) Particle size and the physical properties of growing media for containers. Comm. Soil Sci. Plant Anal 14:209-222

Herrera F, J F Castillo, A F Chica, L Bellido-López (2008) Use of municipal solid waste compost (MSWC) as a growing medium in the nursery production of tomato plants. Biores. Technol. 99:287296.

Jiménez R, M J Robles, M T Lao, S Jiménez (2000) Caracterización de sustratos con cáscara de almendra para uso en cultivos ornamentales. Acta Hort. 32:333-341.

Konduru S, M Evans, R Stamps R (1999) Coconut husk and processing effects on chemical and physical properties of coconut coir dust. HortScience 34:88-90.

Lax A, A Roing, F Costa (1986) A method for determining the cationexchange capacity of organic materials. Plant and Soil 94:349-355.

MAPA (1992-2002) Anuarios del Ministerio de Agricultura, Pesca y Alimentación. España. Disponible en: http://www.mapa. es/es/agricultura/agricultura.htm (julio 2008).

Martínez P F (2000) Presente y futuro de los sustratos en la horticultura mediterránea. Acta Hort. 32:19-31.

Marfá O, A Martínez, R Orozco, L Serrano, F X Martínez (1993) The use of fine grade perlites in lecctuce bag cultures. II Physical properties, rheologic effects and productivity. Acta Hort. $\quad 342$ : 339-347.

Martínez F X (1992) Propuesta de metodología para la determinación de las propiedades físicas de los sustratos. Acta Hort. 11:55-66.

Martínez P, M Abad (1992) Soilless culture of tomato in different mineral substrates. Acta Hort. 323:330-340. 

Muro J, I Irigoyen, P Samiter, P Mazuela, M C Salas, J Soler, M Urrestarazu (2005) Wober fiber as growing medium in hydroponic crop. Acta Hort. 697:179-185.

Noguera P, M Abad, R Puchades, V Noguera, A Maquieira, J Martínez (1997) Physical and chemical properties of coir waste and their relation to plant growth. Acta Hort. 517:279-286.

Puustjarvi V (1994) La turba y su manejo en la horticultura. Ediciones de Horticultura. S. L. Reus. España. 240 p.

Puustjarvi V, R A Roberston (1975) Physical and chemical properties. In: Peat in Horticulture. D W Robinson, J G D Lamb (eds). Academic Press, New York, USA. pp:23-38.

Richards D, M Lane, D V Beardsell (1986) The influence of particlesize distribution in pinebark: sand: brown coal potting mixes on water supply, aeration and plant growth. Sci. Hort. 29:1-14.
Statistical Graphics Corp. (1999) STATGRAPHICS plus for Windows 4.1. Statistical Graphics Corp., Rockville, MD.

Urrestarazu M, G A Martínez, M C Salas (2004) Almond sell waste: Possible local rockwool substitute in soilless crop culture. Sci. Hort. 103:453-460.

Urrestarazu M, M C Salas (2002) El papel de los cultivos sin suelo en la moderna agronomía. Vida Rural 145:54-58.

Verdonck O (1983) Reviewing and evaluation of new materials used as substrates. Acta Hort.150:467-473.

Valera D L, F Molina, J Gil (1999) Los Invernaderos de Almería: Tipología y Mecanización del Clima. Universidad de Almería, España. $278 \mathrm{p}$

Warncke D D (1986) Analyzing greenhouse growth media by the saturation extraction method. HortScience 21:223-225. 